(C) 2007 IEEE. Personal use of this material is permitted. Permission from IEEE must be obtained for all other uses, in any current or future media, including reprinting/republishing this material for advertising or promotional purposes, creating new collective works, for resale or redistribution to servers or lists, or reuse of any copyrighted component of this work in other works. 


\title{
A Geometric Approach with Stability for Two-Dimensional Systems
}

\author{
Lorenzo Ntogramatzidis, Michael Cantoni and Ran Yang
}

\begin{abstract}
In this paper we consider the problem of internal and external stabilisation of controlled invariant and output nulling subspaces via static feedback, for 2-D FornasiniMarchesini models. A computationally tractable procedure for the stabilisation of these subspaces is developed via linear matrix inequality (LMI) techniques. This is a preliminary step towards the solution of so-called disturbance decoupling problems with stability requirements.
\end{abstract}

\section{INTRODUCTION}

The notion of controlled invariance introduced by Basile and Marro in [1] consitutes the key tool of the so-called geometric approach to control theory for LTI systems. The most celebrated control application of this concept is the disturbance decoupling problem, solved for the first time in [1]. The disturbance decoupling problem with the extra requirement of internal stability of the closed-loop was taken into account by Wonham and Morse in [14] via the introduction of $(A, B)$ stabilizability subspaces. An improved solution to the same problem was suggested by Basile and Marro in [2], relying on the concept of self-bounded controlled invariance to avoid eigenspace computation, so that the maximum number of eigenvalues of the closed-loop can be freely placed, [11].

In the last two decades, many valuable results have been achieved in the attempt to develop a geometric theory for 2-D systems, [3], [8], [9], [12]. In particular, in [3] a definition of controlled invariance was proposed for Fornasini-Marchesini (FM) models. This definition, even though less powerful than its 1-D counterpart, enjoys properties that are useful in synthesis problems. In the same paper, it is shown how to employ this notion for the solution of 2-D decoupling problems of nonmeasurable and measurable disturbances without stability requirements. The lack of stability in the solutions of such problems constitutes the biggest limitation in the application of these techniques to real problems, particularly from the perspective of numerical implementation.

The aim of this paper is therefore to provide a characterisation of the stability of the 2-D invariants introduced in [3]. More precisely, the problem of internal and external stabilisation of controlled invariant and output-nulling subspaces by means of suitable static feedback actions is investigated, and

This work was supported in part by The University of Melbourne (MRGS) and the Australian Research Council (Discovery Project DP0664789).

L. Ntogramatzidis and M. Cantoni are with the Department of Electrical and Electronic Engineering, The University of Melbourne, Parkville VIC 3010, Australia $\{$ Int, m. cantoni\}eee. unimelb.edu.au

R. Yang is with the School of Information Science and Technology, Sun Yat-Sen University, Guangzhou 510275, P.R. China. yangranemail.sysu.edu.cn computationally tractable conditions for the stabilisability of such subspaces are derived in terms of LMIs. Armed with these results, the solution of the two aforementioned disturbace decoupling problems with stability of the closed loop is discussed.

\section{2-D INVARIANT SUBSPACES}

We begin by considering the autonomous FornasiniMarchesini (FM) system

$$
x_{i+1, j+1}=A_{1} x_{i+1, j}+A_{2} x_{i, j+1},
$$

where, for all $i, j, x_{i, j} \in \mathbb{R}^{n}$ is referred to as the local state and where $A_{1}, A_{2} \in \mathbb{R}^{n \times n}$. The subspace $\mathscr{J}$ of $\mathbb{R}^{n}$ is $\left(A_{1}, A_{2}\right)$ invariant if

$$
\left[\begin{array}{l}
A_{1} \\
A_{2}
\end{array}\right] \mathscr{J} \subseteq \mathscr{J} \times \mathscr{J} .
$$

The symbol $\times$ denotes the Cartesian product. It is easy to see that $\mathscr{J}$ is $\left(A_{1}, A_{2}\right)$-invariant if and only if $\mathscr{J}$ is both $A_{1}$-invariant and $A_{2}$-invariant.

Lemma 1: Let $\mathscr{J}$ be an $r$-dimensional subspace of $\mathbb{R}^{n}$ and let $J$ be a basis matrix of $\mathscr{J}$, so that $\mathscr{J}=\mathrm{im} J$. The subspace $\mathscr{J}$ is $\left(A_{1}, A_{2}\right)$-invariant if and only if a matrix $X \in \mathbb{R}^{2 r \times r}$ exists such that

$$
\left[\begin{array}{l}
A_{1} \\
A_{2}
\end{array}\right] J=\left[\begin{array}{cc}
J & 0_{n \times r} \\
0_{n \times r} & J
\end{array}\right] X .
$$

The following theorem is the extension for 2-D systems of a very well-known result on the decomposition of the system matrix associated with invariant subspaces.

Theorem 1: There exists an $r$-dimensional subspace $\mathscr{J} \subseteq$ $\mathbb{R}^{n}$ that is $\left(A_{1}, A_{2}\right)$-invariant if and only if there exists a similarity transformation $T$ in $\mathbb{R}^{n}$ such that

$$
T^{-1} A_{i} T=\left[\begin{array}{cc}
A_{i}^{(1,1)} & A_{i}^{(1,2)} \\
0_{(n-r) \times r} & A_{i}^{(2,2)}
\end{array}\right] \quad \text { for } \quad i=1,2 .
$$

Proof: First notice that $\left(A_{1}, A_{2}\right)$-invariance is a coordinatefree concept. To see this, let $J$ be a basis of $\mathscr{J}$ and $J_{N}$ be the transformed basis in the new set of coordinates defined by an arbitrary similarity transformation $T$ in $\mathbb{R}^{n}$, so that $J_{N}=T^{-1} J$. Since $\mathscr{J}$ is $\left(A_{1}, A_{2}\right)$-invariant, in view of (3) there exist $X_{1} \in \mathbb{R}^{r \times r}$ and $X_{2} \in \mathbb{R}^{r \times r}$ such that $A_{i} J=J X_{i}$ for $i=1,2$, leading to $T^{-1} A_{i} T\left(T^{-1} J\right)=\left(T^{-1} J\right) X_{i}$ for $i=1,2$, which are equivalent to

$$
A_{i}^{\prime} J_{N}=J_{N} X_{i}, \quad \text { for } \quad i=1,2,
$$

where $A_{i}^{\prime} \triangleq T^{-1} A_{i} T$. We are now ready to prove (4). Let $T$ be partitioned as $T=\left[\begin{array}{ll}T_{1} & T_{2}\end{array}\right]$ where the columns of $T_{1}$ span 
$\mathscr{J}$, i.e., $\operatorname{im} T_{1}=\mathscr{J}$. Clearly now $J_{N}=T^{-1} J=\left[\begin{array}{c}I_{r} \\ 0_{(n-r) \times r}\end{array}\right]$. By virtue of (5) we find for $i=1,2$

$$
A_{i}^{\prime} J_{N}=\left[\begin{array}{cc}
A_{i}^{(1,1)} & A_{i}^{(1,2)} \\
A_{i}^{(2,1)} & A_{i}^{(2,2)}
\end{array}\right]\left[\begin{array}{c}
I_{r} \\
0_{(n-r) \times r}
\end{array}\right]=\left[\begin{array}{c}
A_{i}^{(1,1)} \\
A_{i}^{(2,1)}
\end{array}\right]
$$

and $\operatorname{im}\left[\begin{array}{l}A_{i}^{(1,1)} \\ A_{i}^{(2,1)}\end{array}\right] \subseteq \operatorname{im} J_{N}$ if and only if $A_{i}^{(2,1)}=0$.

\section{A. Invariant Subspaces and Local-State Trajectories}

Define for $k \in \mathbb{Z}$ the separation set

$$
\mathfrak{C}_{k} \triangleq\{(i, j) \in \mathbb{Z} \times \mathbb{Z} \mid i+j=k\},
$$

along with the so-called global state on $\mathfrak{C}_{k}$ as

$$
\mathscr{X}_{k} \triangleq\left\{x_{i, j} \in \mathbb{R}^{n} \mid(i, j) \in \mathfrak{C}_{k}\right\}
$$

see [5]. If we assign the local state on $\mathfrak{C}_{0}$, equation (1) uniquely determines $\mathscr{X}_{k}$ for all $k>0$. As such, the boundary conditions typically associated with the FM model (1) are assigned by specifying the local state values over the region $\mathfrak{C}_{0}$. In other words, a boundary condition for (1) is an assignment of the form $x_{i, j}=\hat{x}_{i, j} \in \mathbb{R}^{n}$ for all $(i, j) \in \mathfrak{C}_{0}$. Given a subspace $\mathscr{W}$, we denote by $\mathfrak{S}(\mathscr{W})$ the space of all $\mathscr{W}$-valued sequences. By a $\mathscr{W}$-valued boundary condition we will intend $x_{i, j} \in \mathscr{W}$ for all $(i, j) \in \mathfrak{C}_{0}$.

Lemma 2: Given an $\left(A_{1}, A_{2}\right)$-invariant subspace $\mathscr{J}$ for (1), any $\mathscr{J}$-valued boundary condition gives rise to a local state such that $x_{i, j} \in \mathscr{J}$ for all $i, j$.

Proof: Let us write system (1) in the new set of coordinates described by the similarity transformation $T=\left[\begin{array}{ll}T_{1} & T_{2}\end{array}\right]$ such that $\operatorname{im} T_{1}=\mathscr{J}$. If we partition the local state $x_{i, j}$ comformably with $T$ as $\left[\begin{array}{l}x_{i, j}^{\prime} \\ x_{i, j}^{\prime \prime}\end{array}\right]$, by Theorem 1 it follows that (1) can be written as

$$
\begin{gathered}
x_{i+1, j+1}^{\prime}=A_{1}^{(1,1)} x_{i+1, j}^{\prime}+A_{1}^{(1,2)} x_{i+1, j}^{\prime \prime} \\
+A_{2}^{(1,1)} x_{i, j+1}^{\prime}+A_{2}^{(1,2)} x_{i, j+1}^{\prime \prime}, \\
x_{i+1, j+1}^{\prime \prime}=A_{1}^{(2,2)} x_{i+1, j}^{\prime \prime}+A_{2}^{(2,2)} x_{i, j+1}^{\prime \prime} .
\end{gathered}
$$

Moreover, any given $\mathscr{J}$-valued boundary condition is such that $x_{i, j}^{\prime \prime}=0$ for $(i, j) \in \mathfrak{C}_{0}$. By (7) it also follows that $x_{i, j}^{\prime \prime}=0$ for all $i, j$ such that $i+j \geq 0$, which means that the corresponding local state lies on $\mathscr{J}$, i.e., $x_{i, j} \in \mathscr{J}$ for all $i, j$ such that $i+j \geq 0$.

In the new basis defined by $T$ in Lemma 2, the component $x_{i, j}^{\prime}$ of the local state $x_{i, j}$ represents the projection of $x_{i, j}$ onto the invariant subspace $\mathscr{J}$, while the component $x_{i, j}^{\prime \prime}$ represents the canonical projection of the local state $x_{i, j}$ on the quotient space $\mathbb{R}^{n} / \mathscr{J}$. Thus, we refer to the component $x_{i, j}^{\prime}$ of $x_{i, j}$ as the internal component of the local state (with respect to $\mathscr{J}$ ), and to the component $x_{i, j}^{\prime \prime}$ of $x_{i, j}$ as the external component of the local state (with respect to $\mathscr{J}$ ).

\section{B. Internal and External Stability of Invariant Subspaces}

By defining $\left\|\mathscr{X}_{r}\right\| \triangleq \sup _{n \in \mathbb{Z}}\left\|x_{r-n, n}\right\|$, we recall that system (1) - and therefore, with a slight abuse of nomenclature, the pair $\left(A_{1}, A_{2}\right)$ - is asymptotically stable if assuming $\left\|\mathscr{X}_{0}\right\|$ finite we have that $\left\|\mathscr{X}_{i}\right\|$ goes to zero as $i$ goes to infinity. It is well-known that the pair $\left(A_{1}, A_{2}\right)$ is asymptotically stable if and only if

$$
\operatorname{det}\left(I_{n}-A_{1} z_{2}-A_{2} z_{1}\right) \neq 0 \quad \forall\left(z_{1}, z_{2}\right) \in \mathfrak{P}
$$

where $\mathfrak{P}=\left\{\left(\zeta_{1}, \zeta_{2}\right) \in \mathbb{C} \times \mathbb{C}|| \zeta_{1} \mid \leq 1\right.$ and $\left.\left|\zeta_{2}\right| \leq 1\right\}$ is the unit bidisc, or, equivalently, if and only if $\rho\left(A_{1}+e^{i \theta} A_{2}\right)<1$ for all $\theta \in[0,2 \pi]$, here the symbol $\rho(\cdot)$ denoting the spectral radius, [5]. These conditions are not numerically tractable since they should be checked at infinitely many points. For this reason, many conditions for stability of 2-D systems have been proposed in the last two decades, expressed in terms of Lyapunov equations and/or spectral radius conditions of certain matrices, [6], [7], [4]. In this paper we are particularly interested in simple sufficient stability conditions for FM models expressed in terms of LMIs like the one presented in the following lemma, which is one of the most utilised in analysis and synthesis problems involving FM models.

Lemma 3: (Kar and Sigh, 2003, [7])

The pair $\left(A_{1}, A_{2}\right)$ is asymptotically stable if two symmetric positive definite matrices $P_{1}$ and $P_{2}$ exist such that:

$$
\left[\begin{array}{cc}
P_{1} & 0 \\
0 & P_{2}
\end{array}\right]-\left[\begin{array}{c}
A_{1}^{\top} \\
A_{2}^{\top}
\end{array}\right]\left(P_{1}+P_{2}\right)\left[\begin{array}{cc}
A_{1} & A_{2}
\end{array}\right]>0 .
$$

Now we show that stability of (1) can be "split" into two parts with respect to the invariant subspace $\mathscr{J}$. Consider a basis adapted to $\mathscr{J}$. By (8) it turns out that system (6-7) is asymptotically stable if and only if the two pairs $\left(A_{1}^{(1,1)}, A_{2}^{(1,1)}\right)$ and $\left(A_{1}^{(2,2)}, A_{2}^{(2,2)}\right)$ are asymptotically stable. In this basis, the global state on $\mathscr{X}_{k}$ can be partitioned as $\mathscr{X}_{k}=\mathscr{X}_{k}^{\prime} \times \mathscr{X}_{k}^{\prime \prime}$, where $\mathscr{X}_{k}^{\prime} \triangleq\left\{x_{i, j}^{\prime} \mid(i, j) \in \mathfrak{C}_{k}\right\}$ and $\mathscr{X}_{k}^{\prime \prime} \triangleq$ $\left\{x_{i, j}^{\prime \prime} \mid(i, j) \in \mathfrak{C}_{k}\right\}$. Given a $\mathscr{J}$-valued boundary condition, so that $x_{i, j}^{\prime \prime}=0$ for $(i, j) \in \mathfrak{C}_{0}$ (or, in other words, such that $\mathscr{X}_{0}^{\prime \prime}=0$ ), we find from (7) that $x_{i, j}^{\prime \prime}=0$ for all $i, j$ such that $i+j \geq 0$, so that $\mathscr{X}_{k}^{\prime \prime}=0$ for all $k \geq 0$, and (6) becomes

$$
x_{i+1, j+1}^{\prime}=A_{1}^{(1,1)} x_{i+1, j}^{\prime}+A_{2}^{(1,1)} x_{i, j+1}^{\prime} .
$$

If $\left(A_{1}^{(1,1)}, A_{2}^{(1,1)}\right)$ is asymptotically stable, then not only does the local state $x_{i, j}$ lies on $\mathscr{J}$ for all $i, j$ such that $i+j \geq 0$, but $\left\|\mathscr{X}_{k}^{\prime}\right\|$ goes to zero as $k$ goes to infinity. The $\left(A_{1}, A_{2}\right)$ invariant subspace $\mathscr{J}$ is said to be internally stable if the pair $\left(A_{1}^{(1,1)}, A_{2}^{(1,1)}\right)$ is asymptotically stable.

Lemma 4: Let $\mathscr{J}$ be an $r$-dimensional $\left(A_{1}, A_{2}\right)$-invariant subspace, and let $X=\left[\begin{array}{l}X_{1} \\ X_{2}\end{array}\right] \in \mathbb{R}^{2 r \times r}$ be such that (3) hold. Then $\mathscr{J}$ is internally stable if and only if the pair $\left(X_{1}, X_{2}\right)$ is asymptotically stable.

Proof: With respect to a basis of $\mathbb{R}^{n}$ adapted to $\mathscr{J}$, (3) can be written as

$$
\left[\begin{array}{cc}
A_{i}^{(1,1)} & A_{i}^{(1,2)} \\
0_{(n-r) \times r} & A_{i}^{(2,2)}
\end{array}\right]\left[\begin{array}{c}
I_{r} \\
0_{(n-r) \times r}
\end{array}\right]=\left[\begin{array}{c}
I_{r} \\
0_{(n-r) \times r}
\end{array}\right] X_{i}
$$


for $i=1,2$, so that $A_{1}^{(1,1)}=X_{1}$ and $A_{2}^{(1,1)}=X_{2}$.

Consider a non $\mathscr{J}$-valued boundary condition, so that $\mathscr{X}_{0}^{\prime \prime} \neq 0$, and let $x_{i, j}^{\prime \prime}=\hat{x}_{i, j}^{\prime \prime} \in \mathbb{R}^{n-r}$ for $(i, j) \in \mathfrak{C}_{0}$. The dynamics of $x_{i, j}^{\prime \prime}$ are given by

$$
\begin{aligned}
x_{i+1, j+1}^{\prime \prime} & =A_{1}^{(2,2)} x_{i+1, j}^{\prime \prime}+A_{2}^{(2,2)} x_{i, j+1}^{\prime \prime} \\
x_{i, j}^{\prime \prime} & =\hat{x}_{i, j}^{\prime \prime} \quad \text { for } \quad(i, j) \in \mathfrak{C}_{0} .
\end{aligned}
$$

It follows that $\mathscr{X}_{k}^{\prime \prime}$ converges to zero as $k$ goes to infinity if and only if $\left(A_{1}^{(2,2)}, A_{2}^{(2,2)}\right)$ is asymptotically stable. This means that when $(i, j)$ evolves away from $\mathfrak{C}_{0}$ the local state $x_{i, j}$ converges to $\mathscr{J}$. The $\left(A_{1}, A_{2}\right)$-invariant subspace is said to be externally stable if the pair $\left(A_{1}^{(2,2)}, A_{2}^{(2,2)}\right)$ is asymptotically stable. By virtue of (8), it turns out that (1) is asymptotically stable if and only if any $\left(A_{1}, A_{2}\right)$-invariant subspace $\mathscr{J}$ is both internally and externally stable.

\section{2-D CONTROLLED INVARIANT SUBSPACES}

\section{Consider the Fornasini-Marchesini system}

$$
x_{i+1, j+1}=A_{1} x_{i+1, j}+A_{2} x_{i, j+1}+B_{1} u_{i+1, j}+B_{2} u_{i, j+1},(12
$$

here briefly denoted by $\Sigma_{0}$, where, for all $i, j, x_{i, j} \in \mathbb{R}^{n}$ is the local state, $u_{i, j} \in \mathbb{R}^{m}$ is the input, $A_{k} \in \mathbb{R}^{n \times n}$ and $B_{k} \in \mathbb{R}^{n \times m}$ for $k=1,2$. The boundary conditions associated with $\Sigma_{0}$ can still be assigned by specifying the global state over $\mathfrak{C}_{0}$.

Definition 1: (Conte and Perdon, 1988, [3])

The subspace $\mathscr{V} \subseteq \mathbb{R}^{n}$ is controlled invariant for $\Sigma_{0}$ if

$$
\left[\begin{array}{l}
A_{1} \\
A_{2}
\end{array}\right] \mathscr{V} \subseteq(\mathscr{V} \times \mathscr{V})+\operatorname{im}\left[\begin{array}{l}
B_{1} \\
B_{2}
\end{array}\right]
$$

As in the 1-D case, a controlled invariant subspace $\mathscr{V}$ for $\Sigma_{0}$ is such that (12) admits a solution in $\mathfrak{S}(\mathscr{V})$ for any $\mathscr{V}$-valued boundary condition. Whereas in the 1-D case the converse is true as well, with this definition of controlled invariance the subspace of minimal dimension containing a given sequence satisfying (12) is not necessarily controlled invariant for $\Sigma_{0}$. However, Definition 1 enjoys good feedback properties, as shown for the first time in [3], and briefly recalled in the following two lemmas.

Lemma 5: Let $\mathscr{V}$ be a subspace of $\mathbb{R}^{n}$ and let $V$ be a basis matrix of $\mathscr{V}$. The subspace $\mathscr{V}$ is controlled invariant for $\Sigma_{0}$ if and only if two matrices $\Xi$ and $\Omega$ exist such that

$$
\left[\begin{array}{l}
A_{1} \\
A_{2}
\end{array}\right] V=\left[\begin{array}{ll}
V & 0 \\
0 & V
\end{array}\right] \Xi+\left[\begin{array}{l}
B_{1} \\
B_{2}
\end{array}\right] \Omega .
$$

Proof: The proof follows from Definition 1 on noting that (14) is another way of writing (13).

The set of matrices $\Xi$ and $\Omega$ satisfying the linear equation (14) can be parameterised by

$$
\left[\begin{array}{l}
\Xi \\
\Omega
\end{array}\right]=W^{\dagger}\left[\begin{array}{l}
A_{1} \\
A_{2}
\end{array}\right] V+H K,
$$

where $W \triangleq\left[\begin{array}{lll}V & 0 & B_{1} \\ 0 & V & B_{2}\end{array}\right], H$ is a basis matrix of $\operatorname{ker} W$ and $K$ is an arbitrary matrix of suitable size. The symbol $W^{\dagger}$ denotes the Moore-Penrose pseudoinverse of $W$.

Lemma 6: Let $\mathscr{V}$ be an $r$-dimensional subspace of $\mathbb{R}^{n}$. The subspace $\mathscr{V}$ is controlled invariant for $\Sigma_{0}$ if and only if a matrix $F$ exists such that $\mathscr{V}$ is $\left(A_{1}+B_{1} F, A_{2}+B_{2} F\right)$ invariant, i.e., if and only if there exists $X \in \mathbb{R}^{2 r \times r}$ such that

$$
\left[\begin{array}{c}
A_{1}+B_{1} F \\
A_{2}+B_{2} F
\end{array}\right] V=\left[\begin{array}{cc}
V & 0 \\
0 & V
\end{array}\right] X,
$$

Proof: We can use the result in Lemma 5 and set $F=$ $-\Omega\left(V^{\top} V\right)^{-1} V^{\top}$, where $V$ be a basis matrix of $\mathscr{V}$. For such an $F$ we find that (16) holds with $X=\Xi$.

The set of matrices $F$ such that (16) holds - often referred to as friends of the controlled invariant subspace $\mathscr{V}$ - will be denoted by $\mathfrak{F}_{\Sigma_{0}}(\mathscr{V})$. The controlled invariant subspace $\mathscr{V}$ is said to be internally stabilisable (resp. externally stabilisable) if there exists an $F \in \mathfrak{F}_{\Sigma_{0}}(\mathscr{V})$ such that $\mathscr{V}$ is an internally stable (resp. externally stable) $\left(A_{1}+B_{1} F, A_{2}+\right.$ $B_{2} F$ )-invariant.

Now we are interested in characterising the set $\mathfrak{F}_{\Sigma_{0}}(\mathscr{V})$. In view of Lemma $6, F \in \mathfrak{F}_{\Sigma_{0}}(\mathscr{V})$ if and only if a matrix $X$ exists such that (16) holds. It is easily shown that any $F$ satisfying (16) for some $X$ can be associated with a pair $(\Xi, \Omega)$ satisfying (14): take for example $\Omega=-F V$ and $\Xi=$ $X$. Conversely, given a solution $(\Xi, \Omega)$ of (14), it is always possible to determine an $F \in \mathfrak{F}_{\Sigma_{0}}(\mathscr{V})$ such that (16) holds true with $X=\Xi$, by taking any $F$ such that $\Omega=-F V$. Thus, no generality is lost by writing (16) with $X$ replaced by $\Xi=\left[\begin{array}{l}\Xi_{1} \\ \Xi_{2}\end{array}\right]$, partitioned comformably with $\left[\begin{array}{ll}V & 0 \\ 0 & V\end{array}\right]$. Moreover, the solutions of the linear equation $\Omega=-F V$ may be written in the form

$$
F=F_{0}+\Lambda
$$

where $F_{0}=-\Omega\left(V^{\top} V\right)^{-1} V^{\top}$ and $\Lambda$ is any matrix of suitable size such that $\Lambda V=0$. Thus, the only constraint that $\Lambda$ needs to satisfy to guarantee that $F$ is a friend of $\mathscr{V}$ is that $\operatorname{ker} \Lambda \supseteq \operatorname{im} V$. It is easy to show that $F_{0}$ only affects the dynamics of (12) that are internal to $\mathscr{V}$, while $\Lambda$ only affects the dynamics external to $\mathscr{V}$. To see this, let $\xi=\xi^{\prime}+\xi^{\prime \prime} \in \mathbb{R}^{n}$ be such that $\xi^{\prime} \in \mathscr{V}$ and $\xi^{\prime \prime} \in \operatorname{ker} V^{\top}$. Then

$$
\begin{aligned}
F_{0} \xi & =-\Omega\left(V^{\top} V\right)^{-1} V^{\top} \xi^{\prime}-\Omega\left(V^{\top} V\right)^{-1} V^{\top} \xi^{\prime \prime}=F_{0} \xi^{\prime}, \\
\Lambda \xi & =\Lambda\left(\xi^{\prime}+\xi^{\prime \prime}\right)=\Lambda \xi^{\prime \prime} \quad \text { since } \quad \xi^{\prime} \in \mathscr{V} \subseteq \operatorname{ker} \Lambda .
\end{aligned}
$$

Furthermore, since $\xi^{\prime} \in \mathscr{V}$, there exists a vector $\eta$ such that $\xi^{\prime}=V \eta$, and therefore $F_{0} \xi^{\prime}=-\Omega \eta$. We can write the local state equation of the autonomous system obtained by applying $u_{i, j}=F x_{i, j}$ with $F=F_{0}+\Lambda$ to (12) in the new set of coordinates described by the similarity transformation $T=\left[\begin{array}{ll}T_{1} & T_{2}\end{array}\right]$ such that $\operatorname{im} T_{1}=\mathscr{V}$. This yields

$$
\begin{gathered}
{\left[\begin{array}{c}
x_{i+1, j+1}^{\prime} \\
x_{i+1, j+1}^{\prime \prime}
\end{array}\right]=\left[\begin{array}{cc}
M_{1}^{(1,1)} & M_{1}^{(1,2)} \\
0 & M_{1}^{(2,2)}
\end{array}\right]\left[\begin{array}{c}
x_{i+1, j}^{\prime} \\
x_{i+1, j}^{\prime \prime}
\end{array}\right]} \\
+\left[\begin{array}{cc}
M_{2}^{(1,1)} & M_{2}^{(1,2)} \\
0 & M_{2}^{(2,2)}
\end{array}\right]\left[\begin{array}{c}
x_{i, j+1}^{\prime} \\
x_{i, j+1}^{\prime \prime}
\end{array}\right]
\end{gathered}
$$

where $M_{i} \triangleq A_{i}+B_{i} F$. It turns out that the pair $\left(M_{1}^{(1,1)}, M_{2}^{(1,1)}\right)$ only depends on $F_{0}$ while $\left(M_{1}^{(2,2)}, M_{2}^{(2,2)}\right)$ only depends on $\Lambda$. Therefore, we can separately choose $F_{0}$ and $\Lambda$, so that the first stabilises the pair $\left(M_{1}^{(1,1)}, M_{2}^{(1,1)}\right)$ - to stabilise $\mathscr{V}$ internally - and the second stabilises $\left(M_{1}^{(2,2)}, M_{2}^{(2,2)}\right)$ - 
to stabilise $\mathscr{V}$ externally - without affecting the internal stabilisation achieved in the previous step.

\section{A. Internal stabilisation}

In order to stabilise $\mathscr{V}$ internally, we have to find a matrix $F_{0}$ such that the pair $\left(\Xi_{1}, \Xi_{2}\right)$ in (16) is asymptotically stable, as shown in Lemma 4. Since the only degree of freedom here lies in the choice of $\Omega$, which in turn is given by (15), we find that

- when the nullspace of $W \triangleq\left[\begin{array}{lll}V & 0 & B_{1} \\ 0 & V & B_{2}\end{array}\right]$ is zero, there is only one solution to the linear equation (15), and there is no possibility of modifying the internal dynamics of $\mathscr{V}$.

- when $W$ has non-trivial kernel, we can write (15) as

$$
\left[\begin{array}{l}
\Xi_{1} \\
\Xi_{2} \\
\Omega
\end{array}\right]=\left[\begin{array}{l}
L_{1} \\
L_{2} \\
L_{3}
\end{array}\right]+\left[\begin{array}{l}
H_{1} \\
H_{2} \\
H_{3}
\end{array}\right] K
$$

where $\left[\begin{array}{l}L_{1} \\ L_{2} \\ L_{3}\end{array}\right] \triangleq W^{\dagger}\left[\begin{array}{l}A_{1} \\ A_{2}\end{array}\right] V, \operatorname{im}\left[\begin{array}{l}H_{1} \\ H_{2} \\ H_{3}\end{array}\right]=\operatorname{ker} W$ and $K$ is an arbitrary matrix of suitable size. The problem now reduces to finding a $K$ such that the pair $\left(\Xi_{1}, \Xi_{2}\right)$ is asymptotically stable. In Theorem 2 an easily checkable sufficient condition for internal stabilisability of a controlled invariant subspace is presented in terms of an LMI.

Theorem 2: The controlled invariant subspace $\mathscr{V}$ is internally stabilisable if there exist $M=M^{\top}>0, N=$ $N^{\top}>0$ and $Q$ of suitable dimensions such that

$$
\left[\begin{array}{ccc}
-M & 0 & N L_{1}^{\top}+Q^{\top} H_{1}^{\top} \\
0 & -(N-M) & N L_{2}^{\top}+Q^{\top} H_{2}^{\top} \\
L_{1} N+H_{1} Q & L_{2} N+H_{2} Q & -N
\end{array}\right]<0 .
$$

Given a solution $(M, N, Q)$ of (20), a matrix $K$ such that $\left(\Xi_{1}, \Xi_{2}\right)$ in (19) is asymptotically stable is given by $K=Q N^{-1}$.

Proof: The controlled invariant subspace $\mathscr{V}$ is internally stabilisable if and only if there exist symmetric positive definite matrices $P_{1}$ and $P_{2}$ such that $\left(\Xi_{1}, \Xi_{2}\right)$ satisfy (9) in Lemma 3. Since $\Xi_{i}=L_{i}+H_{i} K(i=1,2)$, this is equivalent to the existence of two symmetric and positive definite matrices $\Phi$ and $\Psi$ such that

$$
\left[\begin{array}{ccc}
-\Phi & 0 & \left(L_{1}+H_{1} K\right)^{\top} \Psi \\
0 & -(\Psi-\Phi) & \left(L_{2}+H_{2} K\right)^{\top} \Psi \\
\Psi\left(L_{1}+H_{1} K\right) & \Psi\left(L_{2}+H_{2} K\right) & -\Psi
\end{array}\right]<0 .
$$

By pre- and post-multiplying this matrix inequality by $\operatorname{diag}\left\{\Psi^{-1}, \Psi^{-1}, \Psi^{-1}\right\}$ and by denoting $M=\Psi^{-1} \Phi \Psi^{-1}$, $N=\Psi^{-1}$, and $Q=K \Psi^{-1}$, then a $K$ such that $\left(\Xi_{1}, \Xi_{2}\right)$ is asymptotically stable can be obtained from the solution $(M, N, Q)$ of the LMI (20) with $K=Q N^{-1}$.

\section{B. External stabilisation}

Given a controlled invariant subspace $\mathscr{V}$ and a corresponding basis matrix $V$, let $(\Xi, \Omega)$ be any solution of (15) and let $F_{0}=-\Omega\left(V^{\top} V\right)^{-1} V^{\top}$. We now consider the possibility of choosing a suitable $\Lambda$ to stabilise $\mathscr{V}$ externally. After the application of the control function $u_{i, j}=\left(F_{0}+\Lambda\right) x_{i, j}$, system (12) can be written as

$$
x_{i+1, j+1}=\left(\widehat{A}_{1}+B_{1} \Lambda\right) x_{i+1, j}+\left(\widehat{A}_{2}+B_{2} \Lambda\right) x_{i, j+1}
$$

and where we have defined $\widehat{A}_{i}=A_{i}+B_{i} F_{0}$. The problem is now finding $\Lambda$ such that $\left(\widehat{A}_{1}+B_{1} \Lambda, \widehat{A}_{2}+B_{2} \Lambda\right)$ is asymptotically stable and $\Lambda V=0$.

Theorem 3: The controlled invariant subspace $\mathscr{V}$ is externally stabilisable if there exist $M=M^{\top}>0, N=N^{\top}>0$, $R=R^{\top}>0$ and $S$ of suitable dimensions such that

$$
\left[\begin{array}{ccc}
-M & 0 & \left(\widehat{A}_{1}+B_{1} S^{\top} Q^{\top}\right)^{\top} \\
0 & -(N-M) & \left(\widehat{A}_{2}+B_{2} S^{\top} Q^{\top}\right)^{\top} \\
\widehat{A}_{1}+B_{1} S^{\top} Q^{\top} & \widehat{A}_{2}+B_{2} S^{\top} Q^{\top} & -R
\end{array}\right]<0
$$

with

$$
N R=I
$$

Proof: The condition $\Lambda V=0$ can also be written as $\operatorname{im} \Lambda^{\top} \subseteq$ $\operatorname{ker} V^{\top}$. Then, consider a basis matrix $Q$ of $\operatorname{ker} V^{\top}$, so that $\operatorname{im} \Lambda^{\top} \subseteq \operatorname{im} Q$. It follows that $\Lambda^{\top}=Q S$ for some matrix $S$, so that $\Lambda=S^{\top} Q^{\top}$. The pair $\left(\widehat{A}_{1}+B_{1} S^{\top} Q^{\top}, \widehat{A}_{2}+B_{2} S^{\top} Q^{\top}\right)$ is asymptotically stable if there exist two symmetric positive definite matrices $M$ and $N$ and a matrix $S$ of suitable dimension such that

$$
\left[\begin{array}{ccc}
-M & 0 & \left(\widehat{A}_{1}+B_{1} S^{\top} Q^{\top}\right)^{\top} \\
0 & -(N-M) & \left(\widehat{A}_{2}+B_{2} S^{\top} Q^{\top}\right)^{\top} \\
\widehat{A}_{1}+B_{1} S^{\top} Q^{\top} & \widehat{A}_{2}+B_{2} S^{\top} Q^{\top} & -N^{-1}
\end{array}\right]<0
$$

which is equivalent to (21) along with condition (22).

In order to solve the inequality (21) with the constraint (22), different techniques may be employed. Here we consider the so-called sequential linear programming matrix method (SLPMM) developed in [10]. To this end, we first notice that condition (22) is satisfied if and only if

$$
\operatorname{Trace}(N R)=n \quad \text { and } \quad\left[\begin{array}{cc}
N & I \\
I & R
\end{array}\right] \geq 0
$$

The problem (21-22) can then be solved with the following algorithm.

Algorithm 1: (Leibfritz, 2001, [10])

Step 1: Check the existence of a pair $(N, R)$ satisfying (21) and (23). If such pair exists, denote it with $\left(N^{0}, R^{0}\right)$.

Step 2: Given $\left(N^{k}, R^{k}\right), k \geq 0$, obtain a solution $(N, R)$ together with $S$, to the convex optimization problem

$$
\begin{aligned}
& \min \operatorname{Trace}\left(N R^{k}+N^{k} R\right) \\
& \text { subject to }(21),(23) .
\end{aligned}
$$

Denote this solution with $\left(N_{T}^{k}, R_{T}^{k}\right)$.

Step 3: If $\left|\operatorname{Trace}\left(N_{T}^{k} R^{k}+N^{k} R_{T}^{k}\right)-2 \cdot \operatorname{Trace}\left(N^{k} R^{k}\right)\right| \leq v$, stop, with $v$ a pre-defined sufficiently small positive scalar.

Step 4: Compute $\alpha \in[0,1]$ by solving

$$
\min _{\alpha \in[0,1]} \operatorname{Trace}\left(\left[N^{k}+\alpha\left(N_{T}^{k}-N^{k}\right)\right]\left[R^{k}+\alpha\left(R_{T}^{k}-R^{k}\right)\right]\right) .
$$


Step 5: Set $N^{k+1}=(1-\alpha) N^{k}+\alpha N_{T}^{k}$ and $R^{k+1}=(1-$ $\alpha) R^{k}+\alpha R_{T}^{k}$, then go to Step 2 .

\section{Example 1: Consider (12) with}

$$
\begin{aligned}
A_{1}= & {\left[\begin{array}{llll}
0 & 0 & 0 & 0 \\
0 & 0 & 0 & 0 \\
0 & 0 & 0 & 0 \\
0 & 0 & -2.5 & 0.5
\end{array}\right], A_{2}=\left[\begin{array}{cccc}
3 & -3.5 & -0.5 & 0 \\
-5 & 1.5 & 0 & 0 \\
0 & 0 & 0 & 0 \\
2.5 & 0 & 0 & 0
\end{array}\right], } \\
B_{1}= & {\left[\begin{array}{cc}
0 & 0 \\
0 & 0 \\
1 & -6 \\
-1 & 0
\end{array}\right], \quad B_{2}=\left[\begin{array}{cc}
-5 & 0 \\
1 & -1 \\
-7 & 0 \\
-9 & 0
\end{array}\right] . }
\end{aligned}
$$

This system does not satisfy the sufficient condition (9) for stability. By denoting with $e_{i}$ the $i$-th vector of the canonical basis of $\mathbb{R}^{4}$, it is easily seen that the subspace $\mathscr{V}=$ $\operatorname{span}\left(e_{2}, e_{3}, e_{4}\right)$ is controlled invariant. Hence, $V=\left[\begin{array}{l}0_{1 \times 3} \\ I_{3 \times 3}\end{array}\right]$ is a basis matrix for $\mathscr{V}$. In this case $W=\left[\begin{array}{lll}V & 0 & B_{1} \\ 0 & V & B_{2}\end{array}\right]$ is singular and $H=\left[\begin{array}{llllllll}0 & 6 & 0 & 1 & 0 & 0 & 0 & 1\end{array}\right]^{\top}$ is a basis matrix of ker $W$. Let first $\left[\begin{array}{l}\Xi \\ \Omega\end{array}\right]=W^{\dagger}\left[\begin{array}{l}A_{1} \\ A_{2}\end{array}\right] V$, which yields a matrix $\Xi=\left[\begin{array}{l}\Xi_{1} \\ \Xi_{2}\end{array}\right]$ such that the pair $\left(\Xi_{1}, \Xi_{2}\right)$ does not satisfy condition (9) for stability. As such, by taking

$$
F_{0}=-\Omega\left(V^{\top} V\right)^{-1} V^{\top}=\left[\begin{array}{cccc}
0 & -0.7 & -0.1 & 0 \\
0 & -0.0895 & -0.0184 & 0
\end{array}\right],
$$

we find that the pair $\left(A_{1}+B_{1} F_{0}, A_{2}+B_{2} F_{0}\right)$ is not necessarily internally stable. By changing coordinates according to the similarity transformation $T=\left[\begin{array}{lll|l}e_{2} & e_{3} & e_{4} & e_{1}\end{array}\right]$ which is adapted to $\mathscr{V}$, we find

$$
T^{-1}\left(A_{1}+B_{1} F_{0}\right) T=\left[\begin{array}{ccc|c}
0 & 0 & 0 & 0 \\
-0.1632 & 0.0105 & 0 & 0 \\
0.7 & -2.4 & 0.5 & 0 \\
\hline 0 & 0 & 0 & 0
\end{array}\right]
$$

and

$$
T^{-1}\left(A_{2}+B_{2} F_{0}\right) T=\left[\begin{array}{ccc|c}
0.8895 & -0.0816 & 0 & -5 \\
4.9 & 0.7 & 0 & 0 \\
6.3 & 0.9 & 0 & 2.5 \\
\hline 0 & 0 & 0 & 3
\end{array}\right],
$$

whose structures display the $\left(A_{1}+B_{1} F_{0}, A_{2}+B_{2} F_{0}\right)$ invariance of $\mathscr{V}$. In order to find an $F_{0}$ which internally stabilises the controlled invariant subspace $\mathscr{V}$, let us condider $\left[\begin{array}{l}\Xi \\ \Omega\end{array}\right]=\left[\begin{array}{lll}V & 0 & B_{1} \\ 0 & V & B_{2}\end{array}\right]^{\dagger}\left[\begin{array}{l}A_{1} \\ A_{2}\end{array}\right] V+H K$ where $H=$ $\left[\begin{array}{llllllll}0 & 6 & 0 & 1 & 0 & 0 & 0 & 1\end{array}\right]^{\top}$. In this case, the LMI (20) is feasible, which implies internal stabilisability of $\mathscr{V}$, and its solution yields $K=\left[\begin{array}{lll}-5.8697 & 0.1389 & -0.0031\end{array}\right]$. By using (15) we find that now the pair $\left(\Xi_{1}, \Xi_{2}\right)$ is asymptotically stable, as it satisfies the stability condition (9). With this choice

$$
F_{0}=-\Omega\left(V^{\top} V\right)^{-1} V^{\top}=\left[\begin{array}{cccc}
0 & -0.7 & -0.1 & 0 \\
0 & 0.8627 & -0.0410 & 0.0005
\end{array}\right]
$$

Now

$T^{-1}\left(A_{1}+B_{1} F_{0}\right) T=\left[\begin{array}{ccc|c}0 & 0 & 0 & 0 \\ -5.8763 & 0.1457 & -0.0031 & 0 \\ 0.7 & -2.4 & 0.5 & 0 \\ \hline 0 & 0 & 0 & 0\end{array}\right]$

and

$T^{-1}\left(A_{2}+B_{2} F_{0}\right) T=\left[\begin{array}{ccc|c}-0.0627 & -0.0590 & -0.0005 & -5 \\ 4.9 & 0.7 & 0 & 0 \\ 6.3 & 0.9 & 0 & 2.5 \\ \hline 0 & 0 & 0 & 3\end{array}\right]$,

evidentiate that the pair $(0,3)$ accounting for the external dynamics of $\mathscr{V}$ has not changed by modifying the feedback $F_{0}$ in order to stabilise the controlled invariant subspace $\mathscr{V}$ internally. Since the pair $(0,3)$ is unstable, our goal now is to stabilise $\mathscr{V}$ externally, by means of a feedback matrix $F=F_{0}+\Lambda$, where $\Lambda V=0$. In this case, Algorithm 1 provides a feasible solution to the external stabilisation problem. By choosing $v=10^{-6}$, after 17447 iterations of Steps 1-3, the matrices $N^{k}$ and $R^{k}$ for which the condition in Step 3 is satisfied are found. With their values it is found that $\operatorname{Trace}\left(N^{k} R^{k}\right) \simeq 4.000023$, and the corresponding solution is

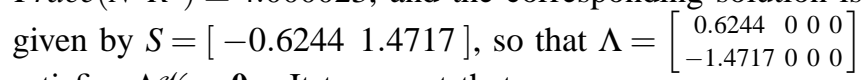
satisfies $\Lambda \mathscr{V}=\mathbf{0}_{m}$. It turns out that

$T^{-1}\left(A_{1}+B_{1} F\right) T=\left[\begin{array}{ccc|c}0 & 0 & 0 & 0 \\ -5.8763 & 0.1457 & -0.0031 & 9.4547 \\ 0.7 & -2.4 & 0.5 & -0.6245 \\ \hline 0 & 0 & 0 & 0\end{array}\right]$

and

$T^{-1}\left(A_{2}+B_{2} F\right) T=\left[\begin{array}{ccc|c}-0.0627 & -0.0590 & -0.0005 & -2.9038 \\ 4.9 & 0.7 & 0 & -4.3715 \\ 6.3 & 0.9 & 0 & -3.1205 \\ \hline 0 & 0 & 0 & -0.1225\end{array}\right]$

are such that the pair accounting for the internal dynamics of $\mathscr{V}$, i.e., $\left(\left[\begin{array}{ccc}0 & 0 & 0 \\ -5.8763 & 0.1457 & -0.0031 \\ 0.7 & -2.4 & 0.5\end{array}\right],\left[\begin{array}{ccc}-0.0627 & -0.0590 & -0.0005 \\ 4.9 & 0.7 & 0 \\ 6.3 & 0.9 & 0\end{array}\right]\right)$, did not change after the introduction of $\Lambda$, so that the internal stabilisation previously performed has not been affected; on the other hand, $\mathscr{V}$ has been externally stabilised since the pair $(0,-0.1225)$ is now asymptotically stable.

\section{OUTPUT-NULLING CONTROLLED INVARIANCE}

In this section we turn our attention to output-nulling subspaces, that are a particular type of controlled invariant subspaces for the FM model $\Sigma$

$$
\begin{aligned}
x_{i+1, j+1} & =A_{1} x_{i+1, j}+A_{2} x_{i, j+1}+B_{1} u_{i+1, j}+B_{2} u_{i, j+1}, \\
y_{i, j} & =C x_{i, j}+D u_{i, j},
\end{aligned}
$$

where $y_{i, j} \in \mathbb{R}^{p}$ is the output vector and the matrices $C$ and $D$ are of suitable dimensions.

The subspace $\mathscr{V} \subseteq \mathbb{R}^{n}$ is an output-nulling controlled invariant subspace for $\Sigma$ if

$$
\left[\begin{array}{c}
A_{1} \\
A_{2} \\
C
\end{array}\right] \mathscr{V} \subseteq\left(\mathscr{V} \times \mathscr{V} \times \mathbf{0}_{p}\right)+\operatorname{im}\left[\begin{array}{c}
B_{1} \\
B_{2} \\
D
\end{array}\right]
$$


see [12]. An output-nulling controlled invariant subspace $\mathscr{V}$ is such that for any $\mathscr{V}$-valued boundary condition, there exists an input function such that the corresponding solution of (24) is in $\mathfrak{S}(\mathscr{V})$ and the corresponding output is zero for all $i, j$ such that $i+j \geq 0$. Such input can always be expressed as a static state feedback. The following lemma summarizes the most important properties of output-nulling subspaces, see [12].

Lemma 7: Let $V$ be a basis matrix of the $r$-dimensional subspace $\mathscr{V}$ of $\mathbb{R}^{n}$. The following statements are equivalent:

- the subspace $\mathscr{V}$ is output-nulling for $\Sigma$.

- A matrix $F \in \mathbb{R}^{m \times n}$ exists such that

$$
\left[\begin{array}{c}
A_{1}+B_{1} F \\
A_{2}+B_{2} F \\
C+D F
\end{array}\right] \mathscr{V}=\left[\begin{array}{cc}
V & 0 \\
0 & V \\
0 & 0
\end{array}\right] X
$$

where $X \in \mathbb{R}^{2 r \times r}$.

The set of output-nulling controlled invariant subspaces of $\Sigma$ is denoted with the symbol $\mathscr{V}(\Sigma)$, and any matrix $F$ such that (26) holds is referred to as an output-nulling friend of $\mathscr{V}$. As in the 1-D case, the set $\mathscr{V}(\Sigma)$ is closed under subspace addition, and the largest output-nulling subspace of $\Sigma$ is denoted by $\mathscr{V}^{\star}$. The subspace $\mathscr{V}^{\star}$ can be computed in finite terms as the $(n-1)$-th term of the monotonically non-increasing subspace of the recurrence

$\mathscr{V}_{i}=\left[\begin{array}{c}A_{1} \\ A_{2} \\ C\end{array}\right]^{-1}\left(\left(\mathscr{V}_{i-1} \times \mathscr{V}_{i-1} \times \mathbf{0}_{p}\right)+\operatorname{im}\left[\begin{array}{c}B_{1} \\ B_{2} \\ D\end{array}\right]\right), \quad \mathscr{V}_{0}=\mathbb{R}^{n}$,

see [3, Proposition 2.7] and [12, Theorem 2]. Due to the invariance property (26), we can introduce the notions of internal stabilisability and external stabilisability for outputnulling controlled invariant subspaces: $\mathscr{V}$ is said to be internally stabilisable (resp. externally stabilisable) if there exists an output-nulling friend $F$ such that $\mathscr{V}$ is an internally stable (resp. externally stable) $\left(A_{1}+B_{1} F, A_{2}+B_{2} F\right)$ invariant. Given a $\mathscr{V}$-valued boundary condition for $\Sigma$, a control function $u_{i, j}=F x_{i, j}$ where $F$ satisfies (26) - i.e. $F$ is an output-nulling friend of $\mathscr{V}-$ is such that $x_{i, j} \in \mathscr{V}$ and $y_{i, j}=0$ for all $i, j$ such that $i+j \geq 0$. To see this, it suffices to substitute $u_{i, j}=F x_{i, j}$ in (24) and observe that when $x_{i+1, j}$ and $x_{i, j+1}$ belong to $\mathscr{V}$, so does $x_{i+1, j+1}$ in view of (26). As a result, for any $\mathscr{V}$-valued boundary condition it is found that $x_{i, j} \in \mathscr{V}$ and $y_{i, j}=0$ since $\mathscr{V} \subseteq \operatorname{ker}(C+D F)$. Hence, the control function maintaining the output at zero and the local state on $\mathscr{V}$ can always be expressed in feeback form. All the material developed in Section III for controlled invariant subspaces can be adapted to output-nulling subspaces with few modifications. Indeed, by substitution of (16) with (26) the stabilisation of output-nulling subspaces via outputnulling static feedback can be carried out along the same lines of the stabilisation of controlled invariant subspaces.

Remark 1: The stabilisation theory developed here for output-nulling subspaces can be used to solve the disturbance decoupling problem with the further requirement of asymptotic stability of the closed loop. A sufficient condition for the solvability of this problem for 2-D systems without stability was first given in [3] in terms of the inclusion of certain subspaces involving $\mathscr{V}^{\star}$, and the feedback matrix $F$ solving the decoupling problem is any output-nulling friend of $\mathscr{V}^{\star}$. However, the application of the control law $u_{i, j}=F x_{i, j}$ does not guarantee asymptotic stability of the closed loop. By adding to this sufficient condition the further condition that $\mathscr{V}^{\star}$ be internally and externally stabilisable, we obtain a set of sufficient conditions for the solvability of the disturbance decoupling with asymptotic stability of the closed loop. In fact, by applying the techniques presented here, in the case where $\mathscr{V}^{\star}$ is internally and externally stabilisable an output-nulling friend $F$ can be found such that the pair $\left(A_{1}+B_{1} F, A_{2}+B_{2} F\right)$ is asymptotically stable.

\section{CONCLUSIONS}

The problem of internal and external stabilisation of controlled invariant and output-nulling subspaces has been considered and solved for the first time for two-dimensional systems. This enables many results on the geometric approach for 2-D systems that have appeared so far in the literature to be improved by adding stability requirements. This obviously extends the applicability of these results in real situations, where due to large bounded frames where the 2-D signals involved are defined and/or for numerical efficiency of the algorithms employed stability may be a necessary and reasonable requirement.

\section{REFERENCES}

[1] G. Basile and G. Marro. Controlled and conditioned invariant subspaces in linear system theory. J. Opt. Theory Appl., 3(5):306$315,1969$.

[2] G. Basile and G. Marro, "Self-bounded controlled invariant subspaces: a straightforward approach to constrained controllability," J. Opt. Theory Appl., vol. 38, no. 1, pp. 71-81, 1982.

[3] G. Conte and A. Perdon. A geometric approach to the theory of 2-D systems. IEEE Trans. Aut. Control, AC-33(10):946-950, 1988.

[4] Y. Ebihara, and Y. Ito, and T. Hagiwara, Exact Stability Analysis of 2-D Systems Using LMIs. IEEE Trans. Aut. Control, AC-51(9):15091513, 2006.

[5] E. Fornasini and G. Marchesini. Doubly-Indexed Dynamical Systems: State-Space Models and Structural Properties. Mathematical System Theory, 12:59-72, 1978.

[6] G.D. Hu and M. Liu. Simple Criteria for Stability of Two-Dimensional Linear Systems. IEEE Trans. Sign. Process., 53(12):4720-4723, 2005.

[7] H. Kar and V. Sigh. Stability of 2-D systems described by the Fornasini-Marchesini first model. IEEE Trans. Sign. Process., 51(6):1675-1676, 2003.

[8] A. Karamanciog̃lu and F.L. Lewis. A geometric approach to 2-D implicit systems. Proc. 29th Conf. on Dec. Contr., Honolulu, Hawaii, December 1990.

[9] A. Karamanciog̃lu and F.L. Lewis. Geometric theory for the singular Roesser model. IEEE Trans. Aut. Control, AC-37(6):801-806, 1992.

[10] F. Leibfritz. An LMI-based algorithm for designing suboptimal static $\mathscr{H}_{2} / \mathscr{H}_{\infty}$ output feedback controllers. SIAM J. Contr. Optim., 39(6):1711-1735, 2001.

[11] M. Malabre, J. Martínez-García, and B. Del-Muro-Cuéllar, "On the fixed poles for disturbance rejection," Automatica, vol. 33, no. 6, pp. 1209-1211, 1997.

[12] L. Ntogramatzidis, and M. Cantoni. New Results and Applications of the Geometric Approach to Two-Dimensional Systems. Proc. 45th Conf. Dec. Contr., San Diego, CA, USA, December 13-15, 2006.

[13] R.P. Roesser. A discrete state-space model for linear image processing. IEEE Trans. Aut. Control, AC-20(1):1-10, 1975.

[14] W.M. Wonham and A.S. Morse. Decoupling and pole assignment in linear multivariable systems: a geometric approach. SIAM J. Contr., 8(1):1-18, 1970. 